\title{
Transparenz normativer Orientierungen in partizipativen TA-Projekten
}

Ein Software-basierter Ansatz

Clemens Mader, Abteilung Technologie und Gesellschaft, Eidgenössiche Materialprüfungs- und Forschungsanstalt (Empa), Lerchenfeldstrasse 5, 9014 St.Gallen (clemens.mader@empa.ch), (1D orcid.org/0000-0001-5366-5354

Lorenz M. Hilty, Institut für Informatik, Universität Zürich (hilty@ifi.uzh.ch), (1) orcid.org/0000-0001-5020-0586

Claudia Som, Abteilung Technologie und Gesellschaft, Empa (claudia.som@empa.ch), (1) orcid.org/0000-0002-8901-4104

Patrick Wäger, Abteilung Technologie und Gesellschaft, Empa (patrick.waeger@empa.ch), (1) orcid.org/0000-0002-2109-6553

In partizipativen TA-Projekten treffen Teilnehmende mit unterschiedlichen normativen Orientierungen aufeinander. Wir stellen einen methodischen Ansatz vor, der alle Beteiligten dabei unterstützen soll, mit dieser Wertepluralität offen und transparent umzugehen, indem die Diskussion in einem Workshop mittels eines Online-Tools zur Befragung der potenziell Teilnehmenden vorbereitet wird. Die „LOTA“ (Landscape of Opinions for Technology Assessment) genannte Methode stützt sich auf globale Ziele, die den Teilnehmenden als normativer Referenzrahmen vorgegeben werden. Diese können Ziele auswählen und priorisieren und auf diese Weise ihre normativen Orientierungen ausdrücken. Das gleichnamige Software-Tool visualisiert eine "Meinungslandschaft" basierend auf den Daten aus der Online-Befragung.

Transparency of normative orientations in participatory TA projects A software-based approach

Participatory TA projects typically bring together people with diverse normative orientations. We present a methodological approach intended to support participants in dealing with the plurality of values in a transparent way by using an online tool to prepare for the discussion. The method called "LOTA" (Landscape of Opinions for Technology Assessment) is based on global goals which are presented to the participants as a normative frame of reference. By prioritizing global goals, the participants can express their normative orientations. The software tool visualizes the "landscape of opinions" resulting from the survey.

Keywords: technology assessment, participation, normative orientation, sustainable development, software

This is an article distributed under the terms of the Creative Commons Attribution License CCBY 4.0 (https://creativecommons.org/licenses/by/4.0/)

https://doi.org/10.14512/tatup.28.1.58

Submitted: 14.11.2018. Peer reviewed. Accepted: 13. 02.2019

\section{Einleitung}

In der Technikfolgenabschätzung (TA) geht es ,um das Spannungsverhältnis zwischen neuen Technikentwicklungen, Gesellschaft und Umwelt" (Open TA-Blog 2018). Bei einem Zeithorizont von mehreren Generationen ist TA zudem im Vorsorgeprinzip verankert und demzufolge der Leitidee der nachhaltigen Entwicklung sehr nahe (Som et al. 2009). TA-Projekte müssen sich aus einer Vielzahl von Gründen mit der normativen Dimension auseinandersetzen (Torgersen 2018). Schon die Wahl eines Zeithorizonts, in dem die Folgen der zur Debatte stehenden Technikentwicklung betrachtet werden, ist eine wertende und damit implizit normative Entscheidung, weil damit zukünftig lebende Menschen als potenziell Betroffene ein- oder ausgeschlossen werden. Ähnliches gilt für die Setzung eines geographischen oder geopolitischen Horizonts in TA-Projekten.

Der Einbezug von gesellschaftlichen Gruppen in die TA kann aus unterschiedlicher Motivation gewählt werden: um Politikberatung zu verbessern und zu bereichern, um zum öffentlichen Dialog beizutragen oder auch um den Prozess des Designs und der Entwicklung der Technik selbst zu unterstützen (Grunwald 2019). Der Fokus der hier vorgestellten Arbeit liegt auf Hilfsmitteln, die dies fördern können, indem sie dabei unterstützen, einen Überblick über die Werthaltungen der Teilnehmenden einer Diskussion zu gewinnen.

In solchen partizipativen TA-Prozessen gelangen Teilnehmende oft zu unterschiedlichen Einschätzungen der Erwünschtheit einer technischen Entwicklung, ohne aber zu verstehen, weshalb sie unterschiedlicher Meinung sind. Fehlt dieses Verständnis, wird eine Chance zur Reflexion des eigenen Standpunktes und damit auch auf eine Annäherung der Standpunkte vertan. Erfahrungsgemäß verfügen Teilnehmende nicht über ein gemeinsames Vokabular, mit dem sie ihre Werthaltungen expli- 
zit ausdrücken könnten. Der hier vorgestellte Ansatz, die LOTA-Methode (Landscape of Opinions for Technology Assessment), beruht deshalb auf der Idee, das Vokabular aus existierenden Dokumenten zu gewinnen, die aus einem breit abgestützten politischen Prozess hervorgegangen sind. Die zwei vielleicht wichtigsten Quellen sind die Allgemeine Erklärung der Menschenrechte und die Ziele für Nachhaltige Entwicklung (SDGs) der Vereinten Nationen.

Unser Ansatz unterscheidet sich von der Multikriteriellen Entscheidungsanalyse (MCDA, z. B. Scholten et al. 2014), obwohl es einige Gemeinsamkeiten gibt. Gemeinsam ist den beiden Ansätzen, dass Wertfragen explizit thematisiert werden, um mit Pluralität konstruktiv umzugehen. Anders als bei MCDA ist es jedoch nicht Ziel von LOTA, Güterabwägungen zu quantifizieren und mittels eines mathematischen Modells Prognosen und Präferenzen zu kombinieren, um robuste Alternativen zu ermitteln, sondern vielmehr eine Diskussion zu fördern, in der ein reflektierteres Verständnis der verschiedenen Positionen wachsen kann und im Idealfall eine Dynamik in Richtung Konsens in Gang kommt.

In diesem Beitrag wird als Beispiel für die Anwendung des methodischen Ansatzes die Technik der autonomen Lieferdrohnen verwendet. Dieses Beispiel, auf Basis eines Testlaufs mit einer Gruppe von Studierenden, dient zur Illustration der Methode.

\section{Partizipation, Werte und Transparenz in der TA}

In den letzten Jahrzehnten wurden verschiedene Methoden für die TA entwickelt oder genutzt, wie Innovative TA (Ropohl 1996), Constructive TA (Schot und Rip 1996), Real Time TA (Guston und Sarewitz 2002), Leitbildorientierte Technikgestaltung (Gleich 2004), Consensus Conferences, Scenario Workshops, Publiforen, Publifocus, Foresight oder Bürgerbeteiligung (Klüver et al. 2000; Joss und Bellucci 2002; Hennen et al. 2004). Diese Ansätze kann man grob in „Experten-TA“ und ,partizipative TA“ einteilen (Sclove 2010). Letztere lassen sich weiter unterscheiden in Verfahren, bei denen Stakeholder einbezogen werden und Verfahren, bei denen die allgemeine Öffentlichkeit einbezogen wird (Bürgerberatung), wenngleich häufig gemischte Formen vorkommen (Grunwald 2019).

Die Folgen einer technischen Entwicklung sollen in einer möglichst frühen Entwicklungsphase abgeschätzt werden (Simonis 2013), damit die Grundlagen und Optionen für informierte Entscheidungen rechtzeitig bekannt sind. Entscheidungen der Weiterentwicklung müssen also unter hoher Unsicherheit getroffen werden und haben in Gesellschaften mit pluralen Wertvorstellungen (Mader und Leitenberger 2016) bei hohem technischem Entwicklungstempo oft weitreichende Folgen (Klüver et al. 2000; Som et al. 2009; Bütschi 2012).

Betrachtet man TA als eine Form der anwendungsorientierten Forschung, so gilt auch hier die Erfahrung aus partizipati- ven Forschungsansätzen, dass Missverständnisse zwischen den verschiedenen teilnehmenden Gruppen entstehen und zu einem Vertrauensverlust führen können. Es ist deshalb wichtig, eine gemeinsame Sprache zu entwickeln, um konstruktiv gemeinsam an Lösungen arbeiten zu können (Engels und Walz 2018). Partizipative Forschung und TA stehen deshalb vor einer doppelten Herausforderung: Sie müssen erstens dem Pluralismus förderliche Bedingungen im Forschungsprozess herstellen und zweitens das Vertrauen in die Forschungsergebnisse wahren, indem sie Transparenz bezüglich Interessen und Werthaltungen der eingebundenen Akteure wie auch der Forschenden sicherstellen. Die Meinungsbilder sollten sowohl in der Forschung als auch unter den teilnehmenden Akteuren transparent gemacht werden, da sie Auswirkungen auf die Ergebnisse der Technikbewertung an sich haben (Kerschner und Ehlers 2016).

\section{Die LOTA-Methodik im TA-Prozess}

„LOTA“ (Landscape of Opinions for Technology Assessment) soll zur Bewältigung der oben beschriebenen Herausforderungen beitragen. LOTA soll den Beteiligten insbesondere helfen zu erkennen, aus welchen Gründen sie die Chancen und Risiken einer Technik unterschiedlich beurteilen. Prinzipiell können diese Gründe den folgenden zwei Kategorien angehören:

1. Die Teilnehmenden gehen von unterschiedlichen deskriptiven Voraussetzungen aus, d. h. sie schätzen die faktischen Möglichkeiten, Grenzen oder die konkrete Ausgestaltung zukünftiger Anwendungen unterschiedlich ein.

2. Die Teilnehmenden gehen von unterschiedlichen normativen Voraussetzungen aus, d.h. sie bewerten die aufgrund von (1) erwarteten Folgen der vorgestellten Technikanwendung in unterschiedlichen Wertesystemen. Dies kann in einem partizipativen Prozess zu situativen Wertungskonflikten führen (Weydner-Volkmann 2019).

Bestandteil von Wertesystemen sind, neben ethischen und rechtlichen, insbesondere moralische Werte, also ,,jene Werte, die den fundamentalen Bedürfnissen und legitimen Erwartungen und Interessen entsprechen, die Menschen (und allenfalls einige Tiere) haben“ (Bleisch und Huppenbauer 2011, S. 42). Das Wertesystem, auf das sich eine Person beim Einschätzen einer Frage von gesellschaftlicher Bedeutung bewusst oder unbewusst bezieht, bezeichnen wir im Folgenden als die „,normative Orientierung" dieser Person.

Die LOTA-Methode unterstützt die Diskussion in partizipativen TA-Projekten, indem sie - in einer dem ersten Zusammentreffen vorgelagerten Online-Befragung - unter anderem die normativen Orientierungen der Teilnehmenden erfasst. Die Ergebnisse werden visualisiert und an die Teilnehmenden zurückzugeben. Die eigentliche Diskussion, die im Rahmen eines Workshops stattfindet, kann somit in Kenntnis der „Meinungslandschaft“ der Teilnehmenden erfolgen. Diese ist dem Konzept 
der „moralischen Landkarte“ (Weydner-Volkmann 2019) verwandt. Indem die Teilnehmenden sehen können, wie sich beispielsweise die an globale Ziele vergebenen Prioritäten in der Gruppe verteilen, werden sie sich der unterschiedlichen normativen Orientierungen in der Gruppe bewusst.

Ein weiterer erwünschter Effekt der vorgelagerten Online-Befragung besteht darin, dass der oder die Moderierende die Daten dazu verwenden kann, nur einen Teil der Befragten für die Teilnahme am Workshop auszuwählen. Dadurch wird es möglich, die Umfrage unter einer wesentlich größeren Zahl von Personen durchzuführen, als für eine gute Interaktion im Workshop wünschbar ist. Mit Hilfe einer Clusteranalyse kann die Moderatorin oder der Moderator Cluster ähnlicher Meinungen bilden und von jedem Cluster eine kleine Zahl an Repräsentierenden (1-3) einladen. Durch diese Heuristik erhöht sich die Chance auf eine reichhaltige Diskussion, in der ein breites Spektrum von Positionen vertreten ist und möglichst alle Argumente zur Sprache kommen.

Bei der Entwicklung der LOTA-Methode war ein zentrales Problem zu lösen: Mit welchem Vokabular, mit welchen Kategorien und im Rahmen welcher normativen Ideale sollen normative Orientierungen explizit erfragt und transparent gemacht werden? Hierfür bieten sich aus der bisherigen Praxis die folgenden Möglichkeiten an:

1. Mit dem Vokabular der Ethik. Diese Option stellt allerdings hohe Anforderungen an die Artikulationsfähigkeit der Teilnehmenden. Außerdem ist es ein weiter Weg, die Implikationen ethischer Positionen für die jeweiligen konkreten Fragen zu analysieren, oder umgekehrt, aus typischen Entscheidungssituationen durch Abstraktion „für ähnliche Entscheidungssituationen wiederkehrende Wertungskonflikte" (Weydner-Volkmann 2019, S. 42) zu gewinnen. Für spezifischere Kontexte mag dies gelingen. Einen interessanten $\mathrm{Zu}-$ gang wählt Vetter (2018) mit der „Matrix for Convivial Technologies". Diese fragt vorgegebene Designkriterien ab, die auf einer bestimmten normativen Position - der Ethik der Konvivialität - beruhen und diese konkretisieren.

2. In psychologischen Kategorien. In diesem Fall könnten subjektive Werthaltungen der Teilnehmenden mit existierenden psychologischen Tests erhoben werden. Eine solche „Psychologisierung" normativer Orientierungen - obwohl sie in anderen Verfahren nicht unüblich ist - könnte jedoch die Diskussion über Wertvorstellungen auf eine Diskussion über situative oder individuelle Präferenzen reduzieren. Damit wären moralische Urteile als entscheidender Bestandteil normativer Orientierung nicht von Präferenzen unterschieden. Wer sich zu moralischen Fragen äußert, tut dies in der Überzeugung, damit ein allgemein verbindliches Prinzip zu vertreten, das er selbst dann einhalten müsste, wenn er dabei gegen eigene Präferenzen und Interessen handelte. Gerade deshalb ist die Diskussion normativer Hintergrundannahmen so wichtig für eine ethische Entscheidungsfindung (Bleisch und Huppenbauer 2011). Nach unserer Auffassung leisten Ansätze, die auf Einstellungen basieren (wie z. B. Kerschner und Ehlers 2016) dies nicht.

3. Durch Bezugnahme auf einen existierenden Rahmen normativer Ideen, die sich international als weitgehend konsensfähig erwiesen haben. Hierzu gehören insbesondere die Allgemeine Erklärung der Menschenrechte (UDHR) und die Ziele für nachhaltige Entwicklung (SDGs) der Vereinten Nationen. Auch weithin verwendete Indikatorensysteme wie der Human Development Index (HDI) oder der Happy Planet Index (HPI) können mit den Kriterien, auf die sie sich stützen, eventuell zu einem gemeinsamen Vokabular beitragen.

Dieser dritte Weg ist jener, den wir mit dem LOTA-Ansatz explorativ beschreiten. Konkret haben wir alle vier oben genannten ,globalen Zielsysteme“ (UDHR, SDGs, HDI, HPI) als Quellen verwendet. Hierbei sei angemerkt:

- Dadurch, dass wir diese Zielsysteme in den Prozess einbringen, werden sie nicht normativ vorausgesetzt. Im Gegenteil: Wir schaffen damit eine Gelegenheit für die Teilnehmenden, sich explizit für oder gegen die darin enthaltenen Ziele zu entscheiden. Beispielsweise können Teilnehmende das globale Ziel „Wohlstand“ in ihre Liste prioritärer Ziele aufnehmen und das Ziel „Gesunde Umwelt“" explizit nicht, oder umgekehrt. Das vorgegebene Zielsystem dient lediglich als „Koordinatensystem“, in dem sich die Teilnehmenden normativ verorten können.

- Dass wir die in den Zielsystemen vorkommenden Themen grundlegend für relevant halten, ist jedoch eine unvermeidbare normative Setzung. Sollte es aber vorkommen, dass Teilnehmende das für sie relevante Thema im gegebenen „Koordinatensystem“ überhaupt nicht vorfinden, besteht die Möglichkeit, ein eigenes Ziel zu formulieren.

- Die Tatsache, dass es sich um globale Ziele handelt, also keine Einschränkung auf nationale oder lokale Kontexte vorgesehen ist, ist ebenfalls eine bewusste normative Setzung, wie in der Einleitung bereits erwähnt. Es ist Teil unseres Ansatzes, TA so zu verstehen, dass die Folgen einer technischen Entwicklung für den ganzen Planeten betrachtet werden sollen.

- Es ist prinzipiell möglich, die Zielsysteme, die wir verwenden, durch andere zu ersetzen und den Ansatz dadurch zu generalisieren.

Die Inhalte der SDGs, der allgemeinen Erklärung der Menschenrechte und die Teilindikatoren für HDI und HPI haben wir nach inhaltlicher Analyse wie folgt zu neun globalen Zielen aggregiert: (1) Sicherung der Grundbedürfnisse; (2) Freiheit und Selbstbestimmung; (3) Sicherheit und Frieden; (4) Gesunde Umwelt; (5) Zugang zu Bildung und Information; (6) Rechtsstaatlichkeit; (7) Gleichheit; (8) Wohlstand; (9) Globaler Konsens und Governance.

Jedes der neun Ziele ist mit einer Sammlung von Auszügen der globalen Zielsysteme in der Originalformulierung hinter- 
legt, auf die sich das jeweilige Oberziel abstützt. Diese Sammlung kann beim Ausfüllen des Fragebogens durch die Teilnehmenden mit einem Mausklick abgerufen werden und dient zur Erläuterung der Oberziele. Die Teilnehmenden können die Liste der Oberziele außerdem individuell ergänzen.

Zur Erhebung normativer Orientierungen wurde ein Fragebogen entwickelt, in welchem die Befragten - unter anderem - aus den genannten globalen Zielen eine Teilmenge auswählen. Anschließend sollen sie die Auswahl nach Priorität ordnen. Es gehört zu der Methode, dass die Teilnehmenden gezwungen werden, selektiv zu sein. Wir hoffen, damit Unterschiede in den normativen Orientierungen zu detektieren. In der derzeitigen Version der Software besteht außerdem ein Zwang, die ausgewählten Ziele in eine strikte Rangordnung zu bringen. Die Erfahrung wird zeigen, ob es eventuell notwendig ist, auch die Gleichwertigkeit von Zielen zuzulassen.

\section{Fallbeispiel Lieferdrohne}

Die LOTA-Methode wird anhand des Fallbeispiels „Lieferdrohne“ in vier Schritten beschrieben. Vorbereitend legt der oder die Moderierende fest, wie viele Ziele die Befragten als Priorität auswählen dürfen. Die Anzahl bestimmt die Komplexität und damit die Dauer des Prozesses. Im folgenden Beispiel konnten Teilnehmende zwischen einem und vier Zielen auswählen.

\section{Schritt 1 (Phase I der Online-Befragung)}

Auswahl und Priorisierung der Ziele, Beantwortung weiterer Fragen zur Einschätzung der Dringlichkeit und langfristigen Gültigkeit der Ziele sowie zur Einschätzung der Chancen und Risiken der zur Diskussion stehenden Technik in Bezug auf die ausgewählten Ziele. Die relativ große „Abstraktionslücke“ zwischen den allgemeinen globalen Zielen und der jeweiligen Technik (hier: Lieferdrohnen) wird durch die individuelle Einschätzung der Teilnehmenden gefüllt. Hier ist bewusst keine Anleitung oder Führung vorgegeben.

\section{Schritt 2 (Auswertung Phase I der Online-Befragung)}

Visualisierung der Ergebnisse der Befragung für jede teilnehmende Person in Radardiagrammen (Abb. 1), wobei die eigene Einschätzung (schwarz) und die durchschnittliche Einschätzung der Teilnehmenden (grün) angezeigt werden; pseudonymisierte Visualisierung der Meinungslandschaft basierend auf einer Clusteranalyse (Abb. 2). Diese Darstellung kann die Moderatorin oder der Moderator z. B. verwenden, um eine Teilmenge der Personen für die Teilnahme am weiteren Prozess auszuwählen. Insbesondere lässt sich die Pluralität der Meinungen in den nachfolgenden Schritten maximieren, indem man von jedem Cluster mindestens eine Person einlädt.

In Abbildung 1 ist zu sehen, dass der Indikator ,Grad der Besorgnis“ bei dieser oder diesem Teilnehmenden in Bezug auf alle von ihr gewählten Ziele leicht höher als im Durchschnitt ist. Ferner erkennt sie Chancen der diskutierten Technik für alle Ziele außer für „Gesunde Umwelt“. Dass bei diesem Ziel der Durchschnittswert jedoch relativ hoch ist gibt bereits Anregun-

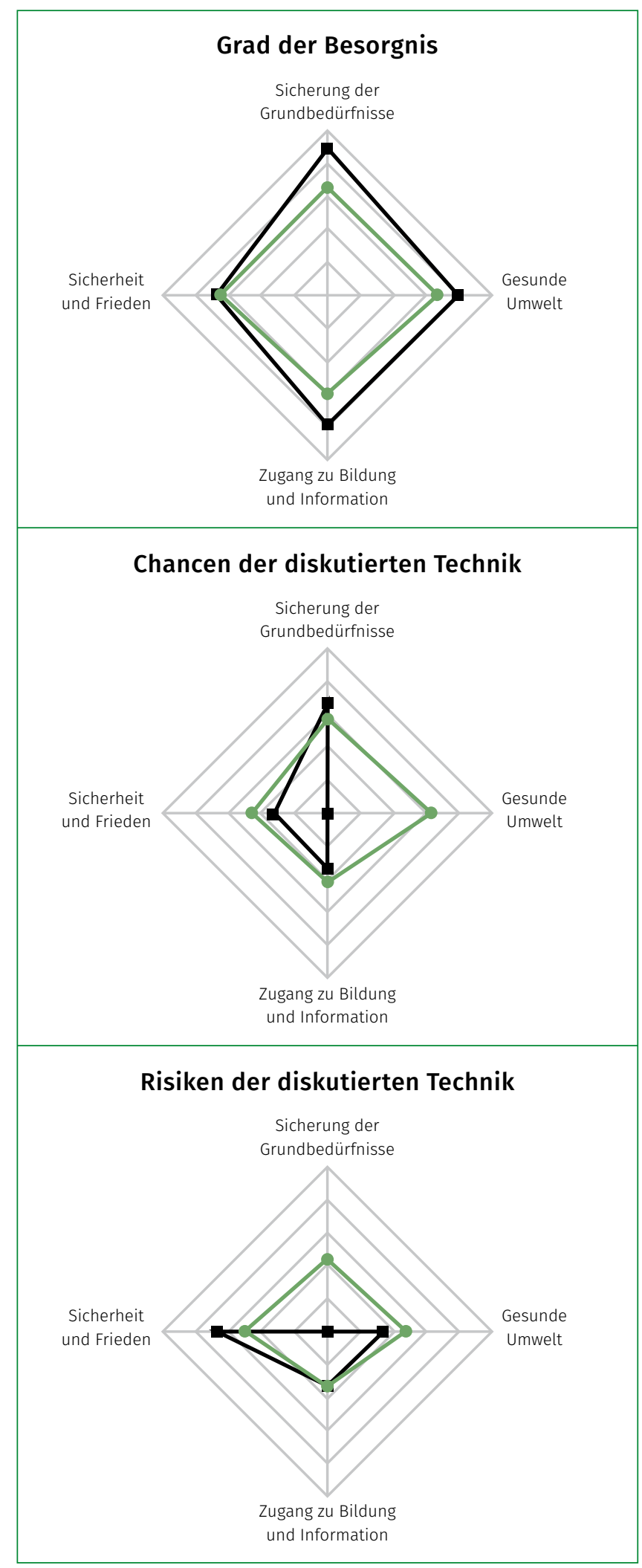

Abb. 1: Radar-Diagramme: zeigen für die ausgewählten Ziele drei Indikatoren, die aufgrund der eigenen Antworten berechnet wurden (schwarz) und den Mittelwert über alle Teilnehmenden (grün) 


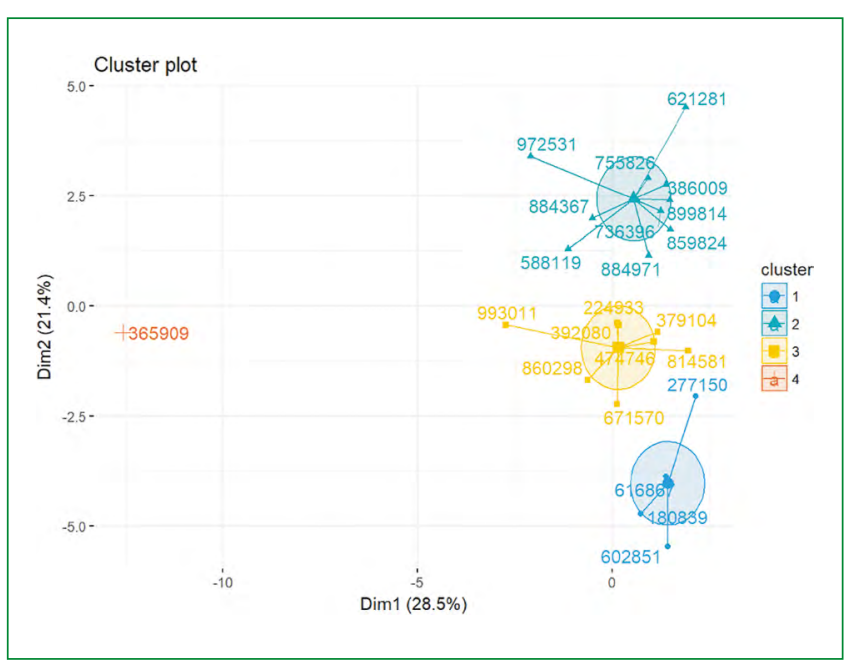

Abb.2: Pseudonymisierte "Meinungslandschaft": Ergebnis der ersten Phase der Online-Befragung. Die sechsstelligen Nummern stehen für Personen und sind nur diesen selbst bekannt. Quelle: Eigene Darstellung

gen für die spätere Diskussion. Ähnlich verhält es sich bei den Risiken, wo diese Person kein Risiko für die „Sicherung der Grundbedürfnisse" sieht, die Gruppe im Durchschnitt dagegen schon.

Abbildung 2 zeigt, wie die Teilnehmenden in einer Clusteranalyse aufgrund ihrer Antworten in Untergruppen eingeteilt werden. Das Verfahren lässt sich grob wie folgt beschreiben: Aufgrund der von ihr gegebenen Antworten kann jede Person als Punkt in einem n-dimensionalen Raum repräsentiert werden. Mit dem K-Means-Algorithmus werden, unter Verwendung der Euklidischen Distanz, ${ }^{1}$ Gruppen bzw. Cluster von möglichst ,ähnlichen“ Personen, also von nahe beieinander liegenden Punkten gebildet. Nur für die visuelle Darstellung der Cluster wird die Anzahl der Dimensionen auf zwei reduziert; die tatsächlichen Distanzen sind in dieser Projektion deshalb verzerrt. Es sind vier Cluster zu sehen, wobei das vierte Cluster (ganz links, orange) nur aus einer einzigen Person mit der Nummer 365909 besteht. Mit dem Ziel, unterschiedliche Positionen in den Diskurs zu integrieren, sollte diese Person aufgrund ihrer zu erwarteten Minderheitsmeinung auf jeden Fall zum Workshop eingeladen werden.

\section{Schritt 3 (Online-Befragung Phase II)}

Das System erstellt eine vereinigte Liste jener ausgewählten Ziele, die die höchste subjektive Relevanz für die Teilnehmenden hatten. Diese Liste ist in der Regel länger als die im Schritt 1 vorgegebene maximale Zahl von Zielen, kann in der Länge aber ebenfalls begrenzt werden. In unserem Beispiel umfasst diese

1 Bei diesem statistischen Verfahren wird aus einer Menge von Objekten eine vorher bekannte Anzahl von k Gruppen gebildet. Der Algorithmus ist eine der am häufigsten verwendeten Techniken zur Gruppierung von Objekten; er bevorzugt Gruppen mit geringer Varianz und ähnlicher Größe. vereinigte Liste sechs Ziele (die später in Abb. 3 zu sehen sind). Die zukünftigen Workshopteilnehmenden werden anschließend auch zu den Zielen befragt, die sie in Phase I selbst nicht ausgewählt hatten, die aber neu in der vereinigten Liste auftreten. Neben den Fragen aus Phase I werden vertiefende Fragen zu den Zielen und ihrer Interaktion (Impact Matrix nach Scholz und Tietje 2002) gestellt, deren Ergebnisse im Schritt 4 als „Impact Graph"visualisiert werden (Abb. 3).

\section{Schritt 4 (Auswertung Phase II der Online-Befragung)}

Visualisierung des Systems von Zielen einschließlich ihrer gegenseitigen Beeinflussung und des Einflusses der diskutierten Technik (Abb. 3). Diese Darstellung liegt zum Auftakt des Workshops schon vor und visualisiert die folgenden Informationen:

Zum einen zeigt die Größe eines Knotens einen Indikator, den wir „Relevanz“ nennen. Er beruht auf einer Aggregation der Antworten auf die folgenden Fragen: Wie wichtig ist dieses Ziel für das Leben der Menschen? Wie lange wird es wichtig sein? Wann werden verheerende negative Folgen auftreten, wenn das Ziel nicht erreicht wird?

Zum anderen zeigen die Pfeile die kausalen Einflüsse zwischen den Zielen und ebenso die Einflüsse der technischen Chancen und Risiken auf die Ziele, wie sie von den Teilnehmenden wahrgenommen werden. Die Dicke eines Pfeils zeigt die Stärke des Einflusses, wobei zunächst bewusst nicht unterschieden wird, ob der angenommene Kausalzusammenhang positiv oder negativ ist.

In dieser dritten Form der Visualisierung werden (im Gegensatz zu Abb. 1 und Abb. 2) nun Mittelwerte über alle Teilnehmenden visualisiert. Die Helligkeit eines Knotens oder Pfeils zeigt zudem die Standardabweichung an: Dunkelgrüne Knoten oder Pfeile zeigen maximale Übereinstimmung an; je heller der Grünton, desto höher die Abweichung unter den Teilnehmenden.

Aus Abbildung 3 lässt sich unter anderem ablesen, dass dem Ziel „Gesunde Umwelt" die höchste Relevanz zugeschrieben wurde, dass die Meinungen hierzu jedoch stark auseinandergehen (hellgrün). „Gleichheit“" bekommt die geringste durchschnittliche Relevanz, hat jedoch den stärksten Einfluss auf „Freiheit und Selbstbestimmung“ und einen deutlichen Einfluss auf „Gesunde Umwelt“: Die Gruppe scheint diesem Ziel eine höhere indirekte als direkte Relevanz zuzuschreiben. Allein das Betrachten der Abhängigkeiten der Ziele untereinander kann interessante Fragen für die Diskussion aufwerfen.

Betrachtet man nun zusätzlich, welchen Einfluss die Teilnehmenden den ,,autonomen Lieferdrohnen“ zuschreiben (repräsentiert durch die horizontalen Pfeile von links und rechts, differenziert nach positivem und negativem Einfluss, d. h. Chancen und Risiken), ergibt sich ein Meinungsbild über die Einschätzung dieser technischen Entwicklung vor dem Hintergrund der globalen Ziele. Im hier gezeigten Fall ist abzulesen, dass relativ einhellig Risiken für „Freiheit und Selbstbestimmung“ gesehen werden. Chancen werden hauptsächlich für den ,Zugang zu Bildung und Information“, zur „Sicherung der Grundbedürfnisse“ und zu „Sicherheit und Frieden“" gesehen. 


\section{Ergebnis}

Indem die Teilnehmenden die Online-Befragung durchlaufen und die visuellen Auswertungen gesehen haben, können sie sich in der Diskussion darauf beziehen. Diese Bezugnahme erleichtert die vertiefende Diskussion insbesondere der normativen Orientierungen, die hinter den Einschätzungen von Chancen und Risiken stehen, damit aber auch die Diskussion deskriptiver Fragen, da diese sich nun klarer von normativen Fragen abgrenzen lassen. Die Rolle von LOTA als Werkzeug ist nach dem Auftakt des Workshops beendet; LOTA ist kein Debattier-Tool, sondern dient als ,Geburtshelfer" für eine transparente, reflektierte Diskussion.

Durch die Visualisierung von Meinungslandschaften hoffen wir, Bewusstsein für die Diversität der Standpunkte und ihren normativen Hintergrund $\mathrm{zu}$ schaffen. Dabei werden die Namen der Personen grundsätzlich nicht verwendet. Es bleibt damit den Teilnehmenden überlassen, ihre Standpunkte in der Diskussion zu erläutern und allenfalls freiwillig aus der Anonymität herauszutreten.

\section{Schlussfolgerungen und Ausblick}

Im Zuge der Forderung an die TA, zu nachhaltiger Entwicklung beizutragen (siehe unter anderem die Konferenzen TA18 und NTA8 im Jahr 2018), werden zugleich die Anforderungen an die Methodik der TA anspruchsvoller. Während es in der transdisziplinären Forschung zur Großen Transformation (WBGU 2016) vorwiegend um die Entwicklung von System-, Ziel- und Transformationswissen für neue Umsetzungsideen und -maßnahmen geht, geht es in der TA um die Abschätzung von Wirkungen von Technikanwendungen. Es ist deshalb wesentlich, ein möglichst breites Spektrum an normativen Orientierungen in TA-Studien einfließen zu lassen. Unser Ansatz kann hier nur einen Baustein beisteuern, ohne den Anspruch zu erheben, alle Aspekte umfassend abzudecken. In einer explorativen Anwendung unter Studierenden haben wir positive Effekte auf die Diskussion beobachtet. Die gegebenen Zielsysteme haben die Teilnehmenden offensichtlich inspiriert, mögliche Anwendungen der diskutierten Technik - hier autonome Lieferdrohnen - in einem breiteren Rahmen zu diskutieren, als dies in konventionellen TA-Workshops üblicherweise der Fall ist. Tests unter den realen Bedingungen eines TA-Projekts sind für das Jahr 2019 geplant.
LOTA soll Geburtshelfer eines pluralistischen und normativ transparenten Diskurses sein und dabei eine globale Perspektive einbringen. Damit trägt die Methode nicht nur zu einer besseren Belastbarkeit der Ergebnisse einer TA bei, sondern unterstützt auch die Selbstreflexion der beteiligten Forschenden, welche in ihrer Rolle als „honest broker“ (Pielke 2007) zusätzlich zu ihrer Rolle als Moderierende auch authentische politische Empfehlungen abgeben sollen. Die Diskussion über mögliche Widersprüche, die hier mitschwingen können, ist nicht neu (Hennen et al. 2004) und soll mit unserem Ansatz nicht ignoriert, sondern im Gegenteil durch den Vorschlag einer neuen Praxis inspiriert werden.

\section{Literatur}

Bleisch, Barbara; Huppenbauer, Markus (2011): Ethische Entscheidungsfindung.

Ein Handbuch für die Praxis. Zürich: Versus Verlag.

Bütschi, Danielle (2012): Knowledge-based policy making. Report of the first parliamentary TA debate held in Copenhagen on June 18, 2012. Bern: TA-Swiss. Online verfügbar unter http://www.pacitaproject.eu/wp-content/ uploads/2012/10/PACITA_4.2_1st-Debatre_report_def_print-version.pdf, zuletzt geprüft am 25. 02.2019.

Engels, Anita; Walz, Kerstin (2018): Dealing with multi-perspectivity in real-world laboratories. In: GAIA 27 (S1), S. 39-45.

Gleich, Arnim von (2004): Leitbildorientierte Technikgestaltung. Nanotechno-

logie zwischen Vision und Wirklichkeit. In: Stefan Böschen, Michael Schneider und Anton Lerf (Hg.): Handeln trotz Nichtwissen. Frankfurt am Main: Campus, S. 159-188. 
Grunwald, Armin (2019): Technology assessment in practice and theory. London: Routledge.

Guston, David; Sarewitz, Daniel (2002): Real-time technology assessment. In: Technology in Society 24, S. 93-109.

Hennen, Leonhard; Petermann, Thomas; Scherz, Constanze (2004): Partizipative Verfahren der Technikfolgen-Abschätzung und parlamentarische Politikberatung. Arbeitsbericht Nr. 96. Berlin: TAB.

Joss, Simon; Bellucci, Sergio (2002): Participatory technology assessment. European perspectives. London: Centre for the Study of Democracy University Westminster.

Kerschner, Christian; Ehlers, Melf-Hinrich (2016): A framework of attitudes towards technology in theory and practice. In: Ecological Economics 126, S. 139-151.

Klüver, Lars et al. (2000): EUROpTA. European participatory technology assessment. Methods in technology assessment and technology decision-making. Kopenhagen: The Danish Board of Technology.

Mader, Clemens; Leitenberger, Anna-Theresa (2016): Relevanz von Stakeholdereinbindung im Nachhaltigkeitsassessment. Die Nachhaltigkeitsprofilmatrix. In: Reinhard Altenburger und Roman Mesicek (Hg.): CSR und Stakeholdermanagement. Wiesbaden: Springer, S. 93-107.

Open TA-Blog (2018): Ein Gespräch mit Dr. Stephan Lingner. Online verfügbar unter https://www.openta.net/blog/ZumTA-VerständnisundderTAPraxisderEAEuropeanAcademy.94, zuletzt geprüft am 14. 01.2019.

Pielke Jr., Roger (2007): The honest broker. Making sense of science in policy and politics. Cambridge: Cambridge University Press.

Ropohl, Günther (1996): Ethik und Technikbewertung. Berlin: Suhrkamp.

Scholten, Lisa; Egger, Christoph; Zheng, Jun; Lienert, Judith (2014): Multikriterielle Entscheidungsanalyse. Neue Ansätze für langfristige Infrastrukturplanung in der Wasserver- und -entsorgung. In: Aqua \& Gas 5, S. 62-69.

Scholz, Roland; Tietje, Olaf (2002): Embedded case study methods. Integrating quantitative and qualitative knowledge. Thousand Oaks, CA: Sage Publications.

Schot, Johan; Rip, Arie (1996): The past and future of constructive TA. In: Technology Forecasting and Social Change 54, S. 251-268.

Sclove, Richard (2010): Reinventing technology assessment. A 215t century model. Washington, DC: Woodrow Wilson International Center for Scholars.

Simonis, Georg (Hg.) (2013): Konzepte und Verfahren der Technikfolgenabschätzung. Wiesbaden: Springer VS.

Som, Claudia; Hilty, Lorenz; Köhler, Andreas (2009): The precautionary principle as a framework for a sustainable information society. Journal of Business Ethics 85 (3), S. 493-505.

Torgersen, Helge (2018): Die verborgene vierte Dimension. In: TATuP - Zeitschrift für Technikfolgenabschätzung in Theorie und Praxis 27 (1), S. 21-27. DOI: 10.14512/tatup.27.1.21.

Vetter, Andrea (2018): The matrix of convivial technologies. Assessing technologies for degrowth. In: Journal of Cleaner Production 197 (2), S. 1619-1886. WBGU (2016): Humanity on the move. Unlocking the transformative power of cities. Berlin: WBGU.

Weydner-Volkmann, Sebastian (2019): Ethische TA als Kartographie situativer Wertungskonflikte. In: TATuP - Zeitschrift für Technikfolgenabschätzung in Theorie und Praxis 28 (1), S. 39-44. D0I: 10.14512/tatup.28.1.39.

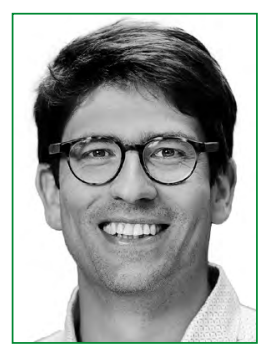

\section{DR. CLEMENS MADER}

ist wissenschaftlicher Mitarbeiter in der Abteilung für Technologie und Gesellschaft der Empa in St. Gallen, sowie im Nachhaltigkeitsteam der Universität Zürich. Er forscht zu Transdisziplinarität, Nachhaltigkeitsprozessen, sowie TA im Bereich Digitalisierung.

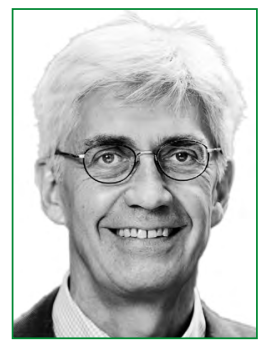

PROF. DR. LORENZ M. HILTY

ist Professor am Institut für Informatik der Universität Zürich und Gruppenleiter in der Abteilung für Technologie und Gesellschaft der Empa in St. Gallen. Seine Forschungsgebiete sind TA im Bereich Digitalisierung sowie IKT-basierte Methoden und Werkzeuge für die Nachhaltigkeitsforschung.

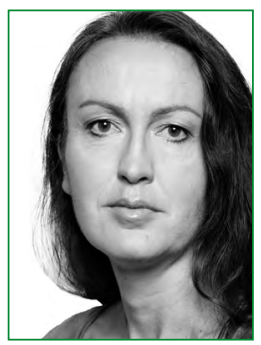

\section{CLAUDIA SOM}

ist Senior Scientist in der Abteilung für Technologie und Gesellschaft der Empa. Sie forscht auf dem Gebiet der TA und nachhaltige Innovation mit Schwerpunkten in den Bereichen Nanotechnologie und Biopolymere.

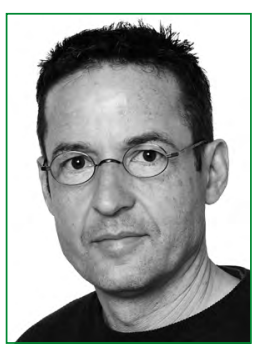

\section{DR. PATRICK WÄGER}

leitet die Abteilung Technologie und Gesellschaft der Empa, welche erforscht, unter welchen Bedingungen neue Materialien und Technologien die Transition zu einer nachhaltigeren Gesellschaft unterstützen können. 